\title{
A Interactive Display Form for Chinese Ink and Wash Painting Using Optical Laser Technique
}

\author{
Yisi Xue \\ Institute of Design and Arts \\ GDUT, Guangzhou, China \\ 153383747@qq.com
}

\author{
Yarong Deng \\ Institute of Design and Arts \\ GDUT, Guangzhou, China \\ 806800257@qq.com
}

\author{
Junming Liang \\ Institute of Design and Arts \\ GDUT, Guangzhou, China \\ 854734252@qq.com
}

\begin{abstract}
Chinese ink and wash painting (CIWP), taking ink as the carrier and based on the variation of the colour of black and white, contains a command of Chinese Humanistic Spirit. However, the reform of new media brings about the innovation of communication mode, cognition and creation. Contemporary CIWP began to change the creative ideas and ways, seeking innovation and change. Especially in the late 20th century, it ushered in a rich cultural breakthrough, which achieved the reform of a series of cultural elements, the renewal of artistic concepts, the transformation of cultural media and the integration of artistic behaviour. The exploration of these aspects has ushered in the unprecedented cross-border study of ink painting art. This paper first analyses the development dilemma of CIWP under the traditional media, and then discusses the innovation brought by the new media to CIWP. Finally, it introduces a reference for the new forms of Contemporary CIWP.
\end{abstract}

Chinese ink painting. Desktop interactive projection. New media. Innovation. Cross-border Cooperation.

\section{INTRODUCTION}

In China in the 1980s, with the reform and opening up and social transformation, culture and art became the pioneers of ideological emancipation. A large number of extraterritorial ideas are introduced through traditional paper media such as books, newspapers, and magazines. For a time, the major "isms" have become the hotspot of social discussion. Chinese traditional art has been greatly impacted by Western art theories.

With this contemporary art movement guided, the value of Chinese traditional culture was shattered by Western modern and contemporary theoretical systems. The break between avant-garde and tradition has also caused reflection of relevant people. Chinese contemporary artists have come out of the aesthetic context of Western "contemporary art" in the 1960s to seek contemporary works of art based on the Chinese stand. The discussion on the innovation of CIWP also moves from the "ink and wash" symbol to the grasp of contemporary art thought and essence.

\section{INNOVATIONS OF CONTEMPORARY CIWP IN THE CONTEXT OF NEW MEDIA}

Art-based media based on the Internet platform gradually replaces traditional media with its fast, open, interactive, and wide-ranging communication characteristics. For CIWP, the renewal of technology and communication is not only related to the change of painting media and painting content, but also plays an important role in finding its own positioning and reconstructing the creative dimension of Chinese ink painting.

Firstly, the new media context creates a personalised and two-way interactive information dissemination environment. Media convergence has made information dissemination and audiences more diverse. On the one hand, it achieves true two-way equality, focusing on the audience's selfselection and multi-differentiation. On the other hand, the public can fully participate in the interaction of artists, new media, and readers, which promotes the transformation of CIWP culture from elite culture to mass culture. 
Secondly, the decentralization of new media context makes the ideas and ways of contemporary art creation more flexible. The meaning of "brush and ink" has been reconstructed by materials and concepts, and the category of CIWP has also been extended. In the face of the global experience of undifferentiated modernity, through the expansion of language, materials and ideas, the expression of contemporary CIWP has been given an equal opportunity to dialogue with others contemporary art.

\subsection{New Life of Contemporary CIWP}

The innovation of art is always accompanied by the advancement of science and technology. Various high-tech means are increasingly being used in the creation and dissemination of art. After entering the era of new media, CIWP have been fully enlarged in the creative space, such as the application in advertising, animation, webpage and film.

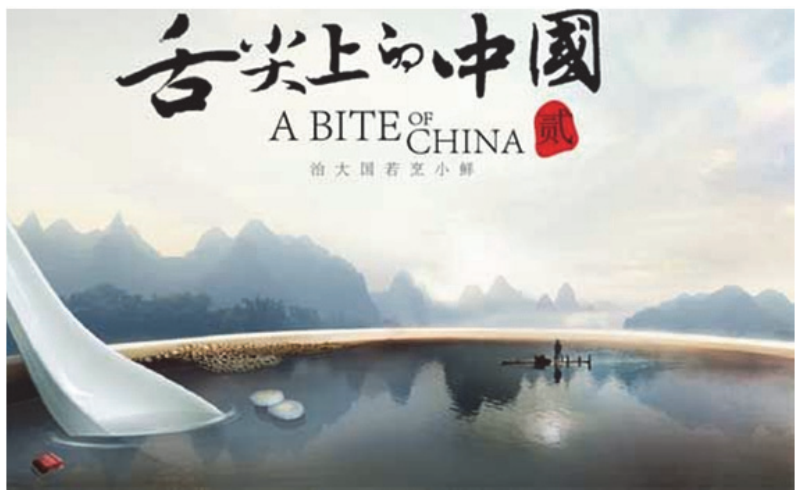

Figure 1: Advertising. "A BITE OF CHINA".

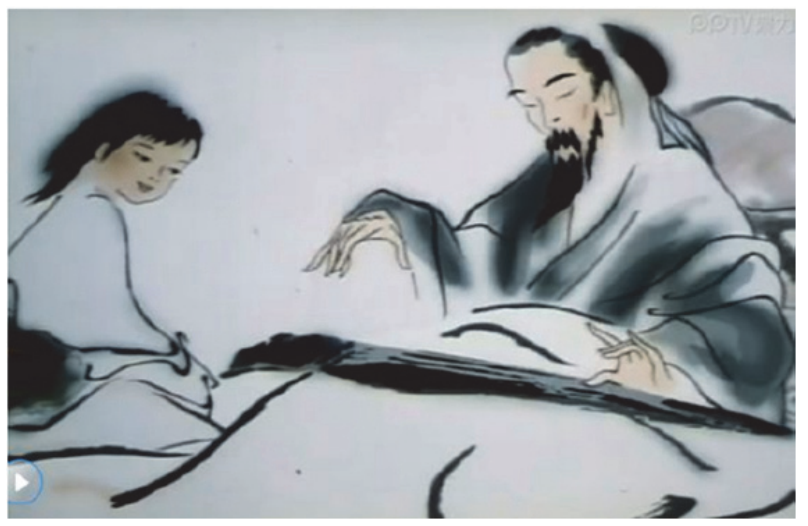

Figure 2: Animation. "Feelings of Mountains and Waters".

The most obvious sign of new media art is connectivity and interactivity. It is this feature that brings more opportunities for the innovation of contemporary Chinese painting, such as the Ink interaction device.

Chinese artist Seph $\mathrm{Li}$ created the interactive installation of digital landscape painting "InkFall". It interacts by capturing the movement of the hand through the camera. When the viewer touches the "painting", the ink is divided by the finger, then the particles of the ink are excited. The shape of each ink line changes according to the direction and speed of the flow, while matching the music of the guqin (a traditional Chinese instrument).

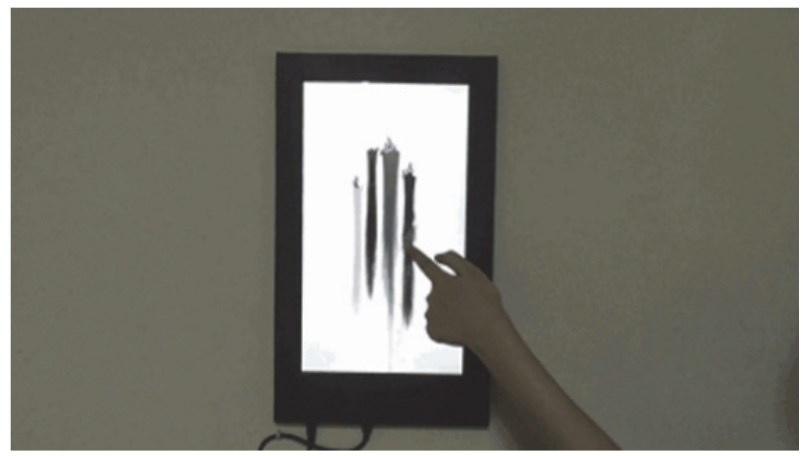

Figure 3: Interactive Implementation. "InkFall"

Artist Ren Min and Dr Hansong Zhang, a scientist from San Francisco Silicon Valley, jointly developed this interactive installation, as shown in Figure 4. The device is based on fluid mechanics and combines a high-performance computer to simulate an ink model. The movement of people watching the exhibition becomes the "creation" variable of the ink-and-wash simulation system, and the viewer becomes the creator with the help of the new media.

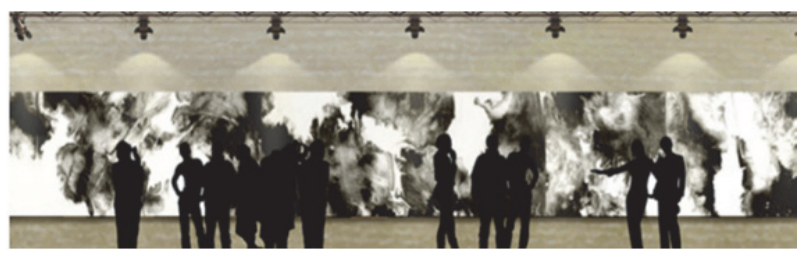

Figure 4: "Ink interaction" display effect.

In fact, the innovation of contemporary Chinese ink painting in the new media era is not just an improvement means for it, but also a new creation of Chinese painting, which has evolved a new artistic ecology. Through the change of creative media, new media art triggers the change of artistic conception space, expression mode and other art experiences that cannot be achieved by traditional media, which causes the artists to think about the history and provides more possibilities for the innovation of contemporary CIWP.

\section{LISTEN TO INK}

"Listen to Ink" was created in response to the China Beijing International Design Week 2018 "Contemporary Ink Design Exhibition" which in order to expand the new thinking of contemporary ink design and explore new ways of contemporary 
ink design to highlight the passion and vitality of contemporary ink painting.

"Listen To Ink" adopts the method of interactive desktop projection, which breaks through the inertia cognition of ink art with visual as the main body. Emphasizing the connection between hearing and ink art, it has realised a new interpretation of ink and ink and expanded new fields. The interaction design of the work is divided into three scenes:

(i) "Ink rhyme feeling":

At the beginning, the slow-moving fishing boats, the waters, the mountains and the lotus bring the interactor into the ink and water perception.

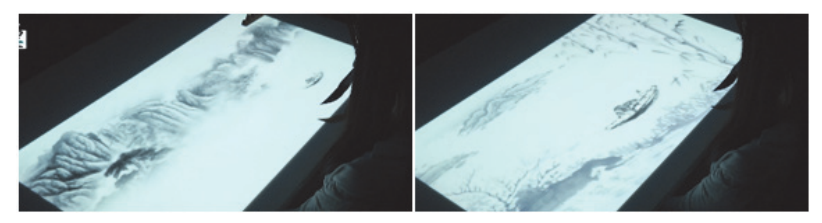

Figure 5: Opening animation.

(ii) "String dialoguing":

The interactor uses the brush to play the strings, then the sounds of the strings are transformed into beating water, rising smoke and flying cranes.

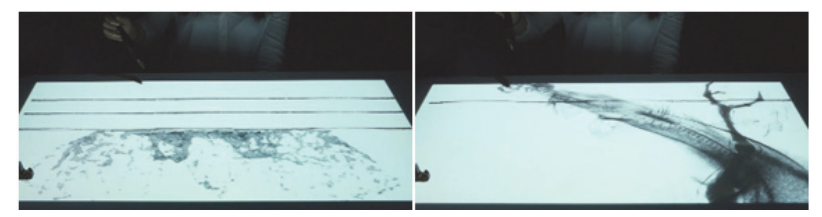

Figure 6: String dialogue.

(iii) "Porcelain fluctuating":

The interactor puts white plates on the table and the patterns are projected accurately onto the white plate along with the change of music. The interactor holds the brush and touches the plate lightly, then ink spots appear, which turn into swimming fish.

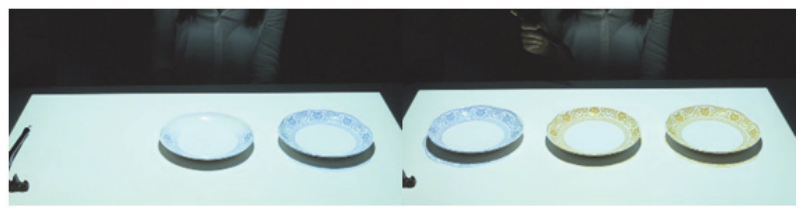

Figure 7: Changing patterns on the plate.

\subsection{Design concept: Cultural connotation of ink art: Unity of nature and man}

Ink art is one of the important carriers for Chinese literati to express their thoughts. The theoretical cornerstone of the birth and development of ink art is Confucianism and Taoism. The Confucianism pursues the artistic creation thought of "cultivating and helping people", and pays special attention to "people". Taoism advocates the idea of "the unity of man and nature" and believes that man is a part of nature and should be integrated with nature.

In summary, in the two thoughts of Confucianism and Taoism, ink painting is the nature, and the audience should be integrated into the nature (ink painting) when viewing. This is the core of the cultural connotation conveyed by the ink art. Therefore, the design concept of "interactive creation between man and ink painting" in this work is exactly coincides with the concept of "harmonious coexistence with nature" pursued by ink painting, so as to inherit the cultural connotation of "unity of nature and man".

\subsection{Design process}

The Instantaneity, entertainment and fragmentation of the public aesthetics in the new media era provided creative ideas to our works. We try to break up and recombine the dull and serious ink art into something that is easy to be understood by the viewers, and complete the interaction by means of playing the guqin in a relaxed and entertaining way. Therefore, ink painting changed from elite culture to mass culture.

\subsubsection{Fragmentation: The regeneration of ink Painting}

In this part, we will break up the Chinese elements contained in the ink paintings to break through the inertia of the people when viewing traditional ink paintings. After investigations and analysis, we extracted representative Chinese elements: Mountain, water, smoke, lotus, bamboo, fish, crane, dragon, fishing boat and guqin.

\subsubsection{Entertainment: The melody of ink painting}

In this part, we will make ink paintings more intimate. We joined the Chinese classical musical instrument guqin, also known as banjos, which was often used by the ancients to express their inner feelings. With a long history and unique timbre, guqin has a familiar Oriental ancient charm for the Chinese people, which is in complete accordance with the temperament of ink painting. With the influence and rendering of this sound, the work strengthens the immersion of viewer's interaction.

\subsubsection{Instantaneity: The vitality of ink painting}

The "instantaneous" aesthetic is the one that the public will hold the initial curiosity about the things that flashed past, such as the app "burning after reading" and "three days visible" for WeChat sharing daily life. This kind of aesthetics allows us to make a thoughtful design in the process of interaction. We want to achieve the aesthetic sensibility of the public by realizing this "instantaneous" content. Therefore, in this project, 
we set up each interactive string for disappearing after playing.

Finally, in order to pursue the artistic conception of ink painting, we do not intend to set the story line. We leave the public with unlimited imagination, hoping that they can put their own thoughts and feelings into this work and interpret it in their own way of thinking and interactive behaviour.

\subsubsection{Cross-border cooperation}

In order to create better visual effects and experience effects, we gathered students majoring in traditional Chinese painting and digital media as well as the computer programming to complete the work of "listen to Ink". Student who major in Chinese Painting provides us with ink painting materials. Then we use PS software to convert them into electronic information and give them dynamic attributes in $A E$, such as boating as shown in Figure 8.

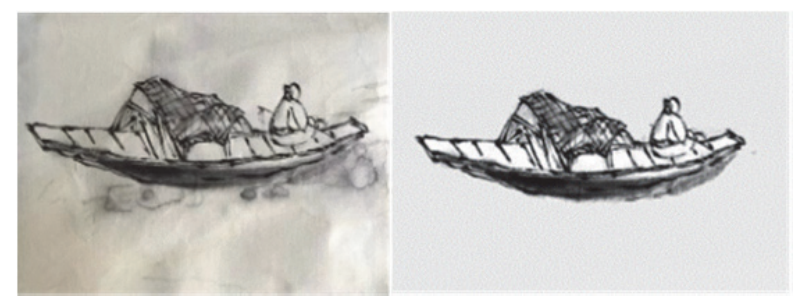

Figure 8: "Boating” drawn by Junming Liang.

In other hand, software such as C4D, AE are used to model and render animated scenes that are difficult to draw by hand: such as smog, flowing water, lotus and bamboo growth. Finally, the completed materials are placed in the PR soundtrack and editing. Once all the interactive materials are ready, we hand it over to the computer team for programming

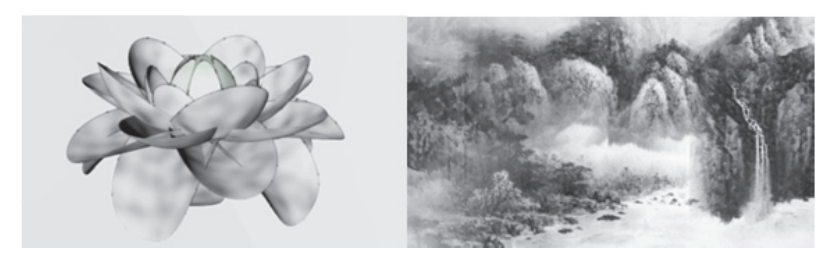

Figure 9: Lotus and mountains rendering.

\subsection{Optical laser technique}

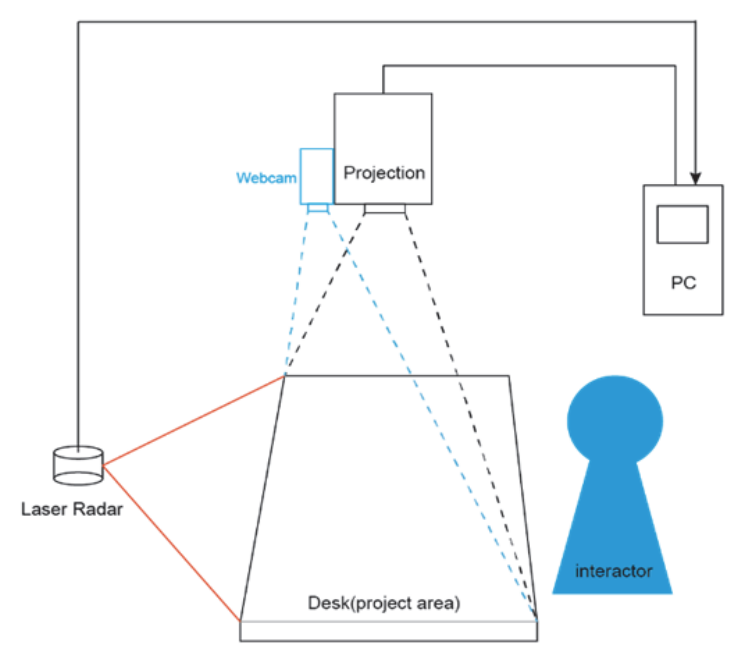

Figure 10: Optical laser system.

The prototype of the proposed infrared optical system is illustrated in Figure 10. This system consists of five things:

- A projector for projecting the interactive video.

- An optical laser radar for detecting the touching point.

- A PC for gathering information and processing data.

- A single webcam is used to locate the edges of desk and capture the moving paths of the brush tip.

- A desk for the experiments.

\subsubsection{Scene one: Ink rhyme introduction}

In this scene, the interactor will tap the screen with a brush. Then, an animation will appear, which brings the interactor into the artistic conception of ink painting. When the brush tip touches the desk, it actives the first touch detection shown in Figure 11 , so the beginning of the ink drawing will follow the brush tip by identifying the coordinate that detected accurately by laser radar.

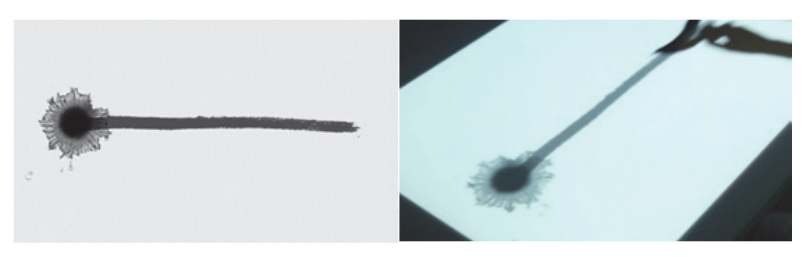

Figure 11: First detection. 


\subsubsection{Scene two: The chord interaction}

In this scene, the interactor will use the brush to pluck the strings, and each string corresponds to a different Chinese element with a different music. In order to do that, we set up a coordinate system. We attach coordinates to each string. By identifying the touch point, we can get the exact location.

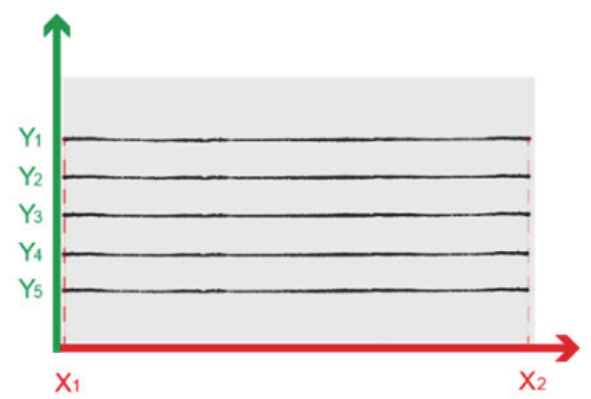

Figure 12: Coordinates for each string.

Suppose the touch point coordinates are $\left(x_{n}, y_{n}\right)$ $x_{1} \leq x_{n} \leq x_{2}$

- When $y_{n}=y_{1}$, it active the animation1 "Rising Smoke".

- When $y_{n}=y_{2}$, it active the animation2 "Flying Crane".

- When $y_{n}=y_{3}$, it active the animation3 "Beating Water".

- When $y_{n}=y_{4}$, it active the animation4 "Swimming Fish".

- When $y_{n}=y_{5}$, it active the animation5 "Flying dragon".

- When all the strings disappear, this interaction ends.

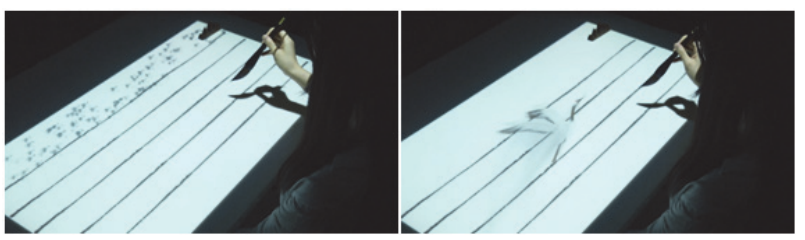

Figure 13: Rising Smoke and Flying Crane.

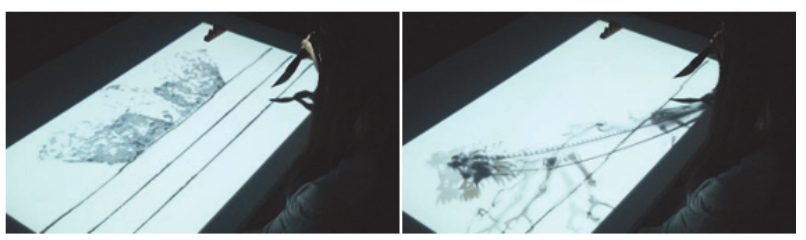

Figure 14: Beating Water and Flying Dragon.

\subsubsection{Scene three: Interact with a fish}

In this scene, the interactor will place plates on the table and click the plate with the brush, and a fish will appear under the pen tip. Click the other plate again, and the fish will jump into the other plate with the pen tip. When the second plate is clicked two times in a row, the interaction ends.
The interactor put the plate on the desk (Note that the plate is equipped with a magnet that can be automatically attached to the specific position), which actives the second touch detection. Meanwhile, the radar captures the positions of the plates, then returns the sensing coordinates to the PC. The approach of how to achieve this interaction is similar to the first one. By identifying the touching point of the brush tip, we can find out the exact coordinate $\left(x_{n}, y_{n}\right)$ again. Note that the coordinates mentioned in this part are not confused with the previous coordinate.

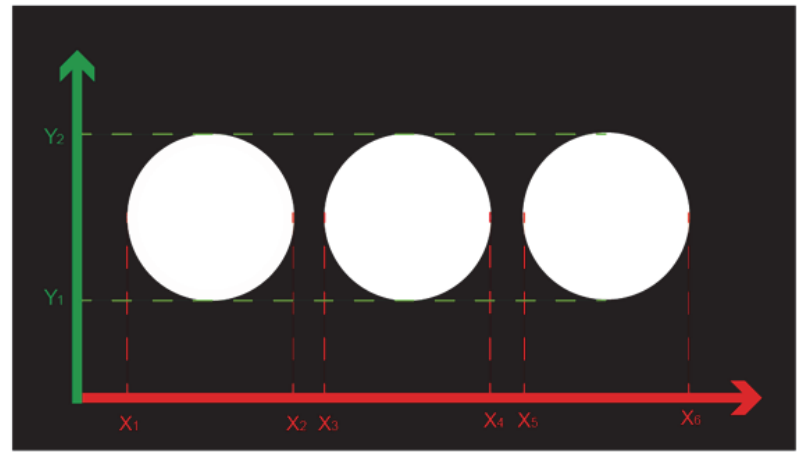

Figure 15: Plates Coordinates.

Suppose that $y_{1} \leq y_{n} \leq y_{2}$. When $x_{1} \leq x_{n} \leq x_{2}$, or $x_{3} \leq x_{n} \leq x_{4}$, or $x_{5} \leq x_{n} \leq x_{6}$, the fish will appear at the point where the brush tip touched, as shown in Figure 15

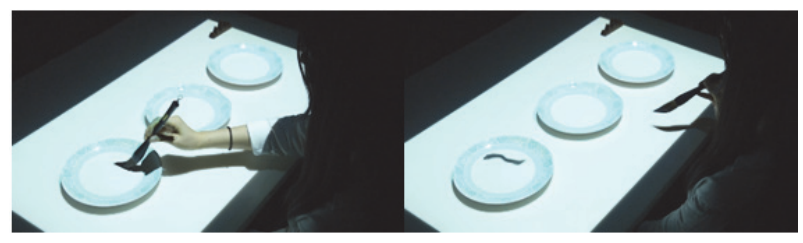

Figure 16: Interact with a fish.

The result of this method breaks through the traditional static perception of ink and wash painting and realises the dynamic audio-visual feast by using the background music made from Chinese traditional musical instrument. This is the Contemporary ink art in line with the public aesthetic taste.

\section{CONCLUSION}

We hope that in the reform and innovation of Chinese painting, we will find the inspection standards of Chinese painting itself, which originate from tradition and transcend tradition.

Contemporary art does not need to adhere to traditional forms in order to maintain its own cultural identity, but it must adapt to the changing times. 
Starting from the essence of Chinese painting, we can avoid the embarrassment of piecing and grafting eastern and western art, and look for the possibility of cross-regional and cross-cultural communication in the new media era. More designs are needed in how this traditional Chinese culture can be best transmitted.

\section{REFERENCES}

Liang, J., Kuan-chin, L., Hsinag-chineh, C. and Wen-june, W. (2018) IEEE ACCESS, 6, pp.1303313040.

Ma, J. (2018) The Change and Unchanging of Contemporary Chinese Painting Innovation under the Evolution of New Media Context. News lovers, 5, pp.57-60.

InkFall (2019)

http://news.ifeng.com/a/20171202/53781370 0.sht

ml (retrieved 2 February 2019).
Kexuan, M. (2018) "Feelings of Mountains and Waters"

http://v.pptv.com/show/QXTZVr4kINI1sz8.html?rcc id=web 2055 (retrieved 2 December 2018).

Welsch, W. (2006) Refactoring Aesthetics. Shanghai Translation Publishing House, Shanghai.

Xiang, X. (2010) Alienated "Decentralization": Examining Electronic Utopia. Nanjing Social Sciences. 10, pp.120-126.

Xiaochun, L. (2008) Research on Contemporary Ink Painting. Shanghai Painting and Calligraphy Publishing House, Shanghai.

Zhang, J., Saboune, A. and El Saddik (2014) 'ransforming a regular screen into a touch screen using a single webcam. J. Display Technol. 10(8), pp.647-659.

Zhang, N. and Zhen, H. (2018) Ink art participated in the applied research of new media creation. This Media.26(03), pp.19-21. 